\title{
Fetal and Neonatal Thrombopoietin Levels in Alloimmune Thrombocytopenia
}

\author{
L. PORCELIJN, C.C. FOLMAN, M. DE HAAS, H.H.H. KANHAI, M.F. MURPHY, \\ A.E.G.KR. VON DEM BORNE, AND J.B. BUSSEL \\ Sanquin Diagnostics, Amsterdam, The Netherlands [L.P., C.C.F., M.d.H.]; Leiden University Medical \\ Center, Leiden, The Netherlands [H.H.H.K.]; John Radcliffe Hospital, Oxford, U.K. [M.F.M.]; Academical \\ Medical Center, Amsterdam, The Netherlands [A.E.G.Kr.v.d.B.]; Weill Medical College of Cornell \\ University, New York, New York, U.S.A. [J.B.B.]
}

\begin{abstract}
ABST
Thrombopoietin (Tpo) is the main hematopoietic growth
factor for platelet production. Plasma Tpo levels in autoimmune
thrombocytopenic patients are normal or slightly elevated. Al-
though thrombocytopenia exists, Tpo levels are not increased
because the produced megakaryocytes and platelets can bind
circulating Tpo, thereby normalizing Tpo levels. In this study,
plasma samples from fetuses and neonates with neonatal alloim-
mune thrombocytopenia (NAIT), a different form of immune
thrombocytopenia, were measured. Umbilical cord samples from
50 fetuses before treatment because of severe thrombocytopenia
and 51 fetuses after treatment, and peripheral blood samples of
21 untreated newborns with NAIT were analyzed. As controls,
plasma Tpo levels were determined in 21 umbilical cord samples
of 14 nonthrombocytopenic fetuses with hemolytic disease re-
sulting from red blood cell alloimmunization and in umbilical
cord samples of 51 healthy newborns. The values were also
compared with the plasma Tpo levels in 193 healthy adults.
\end{abstract}
Mean Tpo levels from the groups of fetuses and neonates, including both NAIT and control plasma, were slightly but significantly elevated compared with levels in healthy adults. Tpo levels in NAIT samples were not significantly different from the levels in hemolytic disease samples or in samples from healthy newborns. Thus, as in autoimmune thrombocytopenic patients, normal Tpo levels are present in NAIT patients.

(Pediatr Res 52: 105-108, 2002)
Abbreviations
HDN, hemolytic disease of the newborn
HPA, human platelet antigens
ITP, idiopathic thrombocytopenic purpura
IVIg, intravenous immunoglobulins
NAIT, neonatal alloimmune thrombocytopenia
Tpo, thrombopoietin

Thrombopoietin is the main hematopoietic growth factor for the megakaryocytic lineage and, therefore, for platelet production. In adults, Tpo is mainly produced by the liver and kidney (1). The concentration of Tpo in blood has been found to depend largely on the number of megakaryocytes in the bone marrow and platelets in the peripheral blood. The plasma level of Tpo is regulated by binding of Tpo to its receptor (mpl), expressed by platelets and megakaryocytes (2-6). After binding, Tpo is eventually destroyed together with the platelets. We and others have shown the diagnostic value of measurement of plasma Tpo levels for discrimination between thrombocytopenia caused by megakaryocyte and platelet production failure (highly elevated Tpo levels) and thrombocytopenia caused by elevated platelet destruction observed in autoimmune thrombocytopenia (normal or only slightly elevated Tpo levels)

Received November 22, 2000; accepted March 15, 2001.

Correspondence: L. Porcelijn, M.D., Platelet/Leukocyte Serology Department, CLB, Sanquin Blood Supply Foundation, Plesmanlaan 125, 1066 CX Amsterdam, The Netherlands; e-mail: 1_Porcelijn@clb.nl
(7-11). In the latter disorder, megakaryocyte numbers in the marrow are normal or increased, as is platelet production. Because sufficient numbers of platelets are produced, even though their destruction may be rapid, Tpo levels are comparable to that in healthy individuals.

NAIT occurs in about 1 in 1000 births and results from maternal alloimmunization against platelet antigens present on fetal platelets but absent on maternal platelets. HPA-1a is the most frequently offending antigen, accounting for approximately $85 \%$ of cases of severe thrombocytopenia. Because of severe thrombocytopenia in utero, in up to $10 \%$ of cases, intracerebral hemorrhage occurs, often leading to severe neurologic sequelae or death (12-14). As in autoimmune thrombocytopenia, in NAIT, fetal platelets are destroyed as a result of antibody binding. Furthermore, it is suggested that reduced megakaryocyte platelet production, or even megakaryocyte destruction, resulting from anti-HPA-1a antibody binding might also contribute to the thrombocytopenia in NAIT patients (15-17). In the current study, Tpo levels were measured in 129 fetal and neonatal NAIT plasma samples and 72 fetal 
and neonatal control plasma samples. We found Tpo levels in fetuses and neonates with NAIT to be not significantly different from the levels in fetal and neonatal controls.

\section{MATERIALS AND METHODS}

Patients. The study was performed with approval of all the involved institutes, and all measurements were performed after informed consent.

Plasma Tpo levels were measured in samples from fetuses with NAIT $(n=113)$, from full-term newborns with NAIT $(n$ $=16$ ), and from nonthrombocytopenic fetuses and neonates, i.e. 21 first or subsequent umbilical cord samples of 14 fetuses with HDN and 51 umbilical cord plasma samples of healthy, full-term newborns (Table 1). The samples were collected in New York (Weill Medical College), Leiden (Leiden University Medical Center), and Oxford (John Radcliffe Hospital). Tpo measurement was performed in the Central Laboratory of the Blood Transfusion Service, Sanquin Diagnostics, Amsterdam. All NAIT cases included in this study involved HPA-1a alloantibodies. Sixteen neonatal samples and 50 umbilical cord samples of 50 fetuses were from NAIT patients who were not treated. Sixty-three umbilical cord samples of 51 fetuses were from NAIT patients who received treatment in utero via administration to their mothers; 40 of these 63 samples were from 34 fetuses who were also included in the untreated group. Treatment consisted of IVIg ( $1 \mathrm{~g} / \mathrm{kg} / \mathrm{wk})$, with or without corticosteroids (prednisone $1 \mathrm{mg} / \mathrm{kg} / \mathrm{d}$ or dexamethasone 1.5 $\mathrm{mg} / \mathrm{d}$ ) administered to the mother during pregnancy and/or intrauterine platelet transfusions administered to the fetus, after umbilical cord sampling. Samples were drawn at $24 \pm 3$ (mean \pm SD) wk of pregnancy for the untreated fetal group, at $33 \pm$ $4 \mathrm{wk}$ of pregnancy for the treated fetal group, at $28 \pm 4 \mathrm{wk}$ of pregnancy for the HDN group, at d 1-7 postpartum for the neonatal NAIT group, and directly postpartum for the neonatal control group. If treatment was necessary and consisted of intrauterine platelet transfusions, samples were drawn before transfusions were given (this was also true for the patients included in the untreated fetal NAIT group). Fetal and neonatal blood samples were aspirated in syringes with EDTA or heparin. Platelet counts were performed with a Coulter counter (Beckman Coulter, Inc., Fullerton, CA, U.S.A.). Methods used for aspiration of samples and for intrauterine platelet transfusions were comparable in the different institutes and have been described previously $(18,19)$. No problems occurred that could be related to the blood sampling.
Plasma Tpo levels of 193 healthy adults for use as controls were measured previously in another study (20).

Tpo ELISA. A solid-phase sandwich ELISA for measurement of plasma Tpo concentrations was performed as previously described (20). Normal Tpo levels, as determined in a population of 193 healthy individuals, were $11 \pm 8$ arbitrary units $(\mathrm{AU}) / \mathrm{mL}$ (range 4-32 AU, 2.5th-97.5th percentile). One AU equals 9 pg of recombinant Tpo (Research Diagnostics, Flanders, NJ, U.S.A.).

Statistical analysis. Statistical analysis was performed in SPSS for Windows, release 6.1.3 (SPSS Inc., Chicago, IL, U.S.A.). For comparison of groups, the Kruskal-Wallis ANOVA and the Mann-Whitney $U /$ Wilcoxon rank sum $\mathrm{W}$ test was used. The correlation between two variables was calculated with Spearman correlation coefficients.

\section{RESULTS}

The platelet count was $68 \pm 28 \times 10^{9} / \mathrm{L}($ mean $\pm \mathrm{SD})$ in the full-term, untreated newborn NAIT group; $37 \pm 34 \times 10^{9} / \mathrm{L}$ in the untreated fetal NAIT group; $76 \pm 61 \times 10^{9} / \mathrm{L}$ in the treated fetal NAIT group; and $190 \pm 53 \times 10^{9} / \mathrm{L}$ in the nonthrombocytopenic hemolytic disease group (Table 1). Platelet counts were not determined in the healthy neonates, but they had no signs or symptoms of bleeding. Further study will be necessary to analyze the influence of treatment on the fetal platelet numbers, but, although the mean platelet numbers in the treated fetal group is significantly higher $(p=0.003$, Table 1$)$ than in the untreated group, the statistical analysis of platelet numbers in fetuses $(n=40)$ included in both the untreated and treated fetal NAIT groups did not show a significant difference $(p=$ 0.06). Plasma Tpo levels were $21 \pm 13 \mathrm{AU} / \mathrm{mL}$ (range 9-47) in the neonatal NAIT samples $(n=16), 26 \pm 18 \mathrm{AU} / \mathrm{mL}$ (range 4-89) in the untreated fetal NAIT samples $(n=50), 26$ $\pm 17 \mathrm{AU} / \mathrm{mL}$ (range 6-97) in the treated fetal NAIT samples ( $n=63$ ), $27 \pm 24$ (range 2-93) in the samples from healthy neonates $(n=51)$, and $18 \pm 8 \mathrm{AU} / \mathrm{mL}$ (range 5-34) in the fetal HDN samples $(n=21)$.

Post hoc testing showed that plasma Tpo levels in the samples of the NAIT neonates and the untreated and the treated NAIT fetuses were comparable with Tpo levels in the samples of the nonthrombocytopenic HDN controls $(p=0.09,0.03$, and 0.8 , respectively) and the healthy neonates ( $p=0.8,0.5$, and 0.3 , respectively) (Fig. 1). The plasma Tpo levels in both the fetal HDN and the healthy newborn controls were slightly, though significantly $(p<0.001)$, higher than plasma Tpo

Table 1. Patient characteristics

\begin{tabular}{|c|c|c|c|c|c|c|}
\hline & \multirow{2}{*}{$\begin{array}{c}\text { Number of } \\
\text { samples }\end{array}$} & \multirow{2}{*}{$\begin{array}{c}\text { Number of } \\
\text { patients }\end{array}$} & \multirow{2}{*}{$\begin{array}{c}\text { Gestational } \\
\text { age (wk) } \\
\text { (mean } \pm \mathrm{SD})\end{array}$} & \multirow{2}{*}{$\begin{array}{l}\text { Platelet count } \\
\left(\times 10^{9} / \mathrm{L}\right) \\
(\text { mean } \pm \mathrm{SD})\end{array}$} & \multicolumn{2}{|c|}{ Plasma Tpo levels (mean $\pm \mathrm{SD})$} \\
\hline & & & & & $(\mathrm{AU} / \mathrm{mL})$ & $(\mathrm{pg} / \mathrm{mL})$ \\
\hline Untreated fetal NAIT & 50 & 50 & $24 \pm 3$ & $37 \pm 34$ & $26 \pm 18$ & $234 \pm 162$ \\
\hline Treated fetal NAIT & 63 & 51 & $33 \pm 4$ & $76 \pm 61$ & $26 \pm 17$ & $234 \pm 153$ \\
\hline Full-term newborns NAIT & 16 & 16 & 1-7 days p.p. & $68 \pm 28$ & $21 \pm 13$ & $189 \pm 117$ \\
\hline Control fetuses (HDN) & 21 & 14 & $28 \pm 4$ & $190 \pm 53$ & $18 \pm 8$ & $162 \pm 72$ \\
\hline Healthy full-term newborns & 51 & 51 & Directly PP & NT & $27 \pm 24$ & $243 \pm 216$ \\
\hline Healthy adults & 193 & 193 & $38 \pm 11 *$ & $150-450$ & $11 \pm 8$ & $99 \pm 72$ \\
\hline
\end{tabular}

PP, postpartum; NT, not tested.

* Age in years. 
levels in healthy adults. The same held true when comparing the Tpo values of the NAIT neonates and the treated and untreated NAIT fetuses with the Tpo levels in healthy adults ( $p$ $<0.001)$. No differences in Tpo plasma levels were detected comparing plasma samples from fetuses with NAIT before and after treatment. No statistically significant correlation was detected between platelet numbers and Tpo levels, either in the total NAIT group (Fig. 2) or in the separate groups or between Tpo levels and age at the time of blood sampling. Furthermore, the anticoagulant used during blood collection or the institute at which blood was drawn did not influence the test results (data not shown).

\section{DISCUSSION}

Screening for NAIT is not routinely performed, and treatment of the initial case in a family mostly consists of postnatal treatment with IVIg and/or platelet transfusions (negative for the antigen involved). In subsequent pregnancies, treatment of the mother with IVIg and corticosteroids and of the fetus with intrauterine platelet transfusions is possible and often used. Trials are in progress to clarify the relative merits of each form of treatment, although, increasingly, it is desirable to avoid fetal sampling, if feasible. We and others detected normal or only slightly elevated Tpo levels in ITP patients (7-10). We now find that Tpo levels in NAIT fetuses are also normal or only slightly elevated compared with levels in nonthrombocytopenic age-matched controls. Thus, as in ITP, in alloimmunemediated thrombocytopenia it appears that normal Tpo removal occurs, resulting in normal levels being maintained in the circulation. This indicates that there is predominantly normal platelet production in fetuses and newborns with NAIT. It seems to contradict the hypothesis that the anti-HPA-1a antibodies would bind to glycoprotein IIb/IIIa on megakaryocytes and either inhibit thrombocytopoiesis or destroy them outright (15-17). We, like others $(21,22)$, found slightly but significantly higher Tpo levels in fetal/neonatal plasma com-

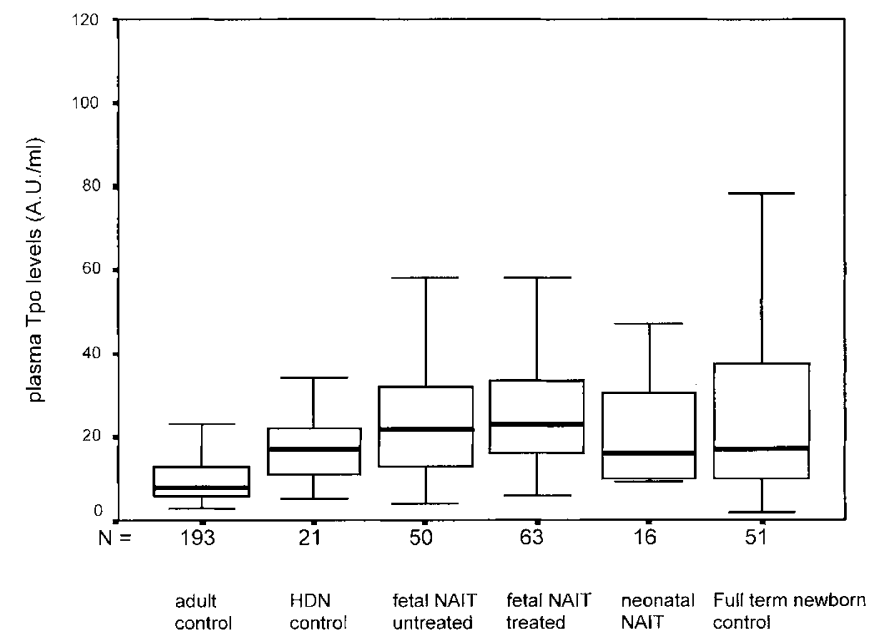

Figure 1. Box plots of Tpo levels in NAIT and controls. Box plots represent the interquartile range that contains $50 \%$ of the values. The whiskers are lines that extend from the box to the highest and lowest values, excluding outliers. A line across the box indicates the median. Tpo values of all separate groups were elevated compared with the adult controls; $p<0.001$.

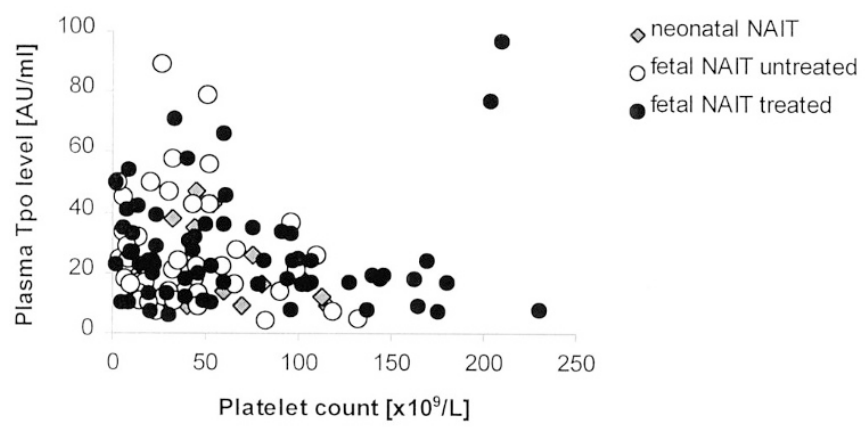

Figure 2. Platelet counts vs plasma Tpo levels in NAIT. Platelet counts and plasma Tpo levels are not correlated. Tpo levels are comparable in the different groups $(p>0.1)$, with or without the two outliers. The two outliers cannot be explained by differences in clinical data, treatment, or sampling procedures. Perhaps Tpo release from platelets occurred in vitro as a result of some unwanted platelet clotting in the EDTA samples.

pared with plasma from adults (Fig. 1). For the slightly elevated Tpo levels in fetuses/neonates (also with normal platelet counts), several explanations are possible-e.g. an increased production of Tpo, considering that in fetuses and neonates the spleen may also contribute to Tpo production (23); less expression of Tpo receptors on megakaryocytes in neonates, as described by Kuwaki et al. (24), resulting in slower removal; or an increased constitutive production of Tpo, inasmuch as the rate of growth of the fetus as well as the neonate is remarkably high, and, therefore, there is a continuous requirement for blood elements for neovasculature. Further study is necessary to explain the higher Tpo levels in neonates compared with adult levels.

The down-regulation of Tpo plasma levels by platelets appears to prevent an increase in Tpo levels in all cases in which thrombocytopenia is caused by increased platelet destruction. Thus, the fact that, as in ITP patients, Tpo levels in NAIT patients are not increased or are only marginally increased may have important therapeutic implications. Injection of Tpo, or Tpo-mimicking peptide, could likely be applied to increase platelet production and platelet count. This approach has been shown to be effective in chimpanzees with HIV-ITP (25) and in a few patients (26).

\section{REFERENCES}

1. Kaushansky K 1998 Thrombopoietin. N Engl J Med 339:746-754

2. Debili N, Wendling F, Cosman D, Titeux M, Florindo C, Dusanter-Fourt I, Schooley K, Methia N, Charon M, Nador R et al. 1995 The Mpl receptor is expressed in the megakaryocytic lineage from late progenitors to platelets. Blood 85:391-401

3. Broudy VC, Lin NL, Sabath DF, Papayannopoulou T, Kaushansky K 1997 Human platelets display high affinity receptors for thrombopoietin. Blood 89:1896-1904

4. Folman CC, de Jong SCM, de Haas M, von dem Borne AEGKr 2001 Analysis of the kinetics of thrombopoietin uptake during platelet transfusion. Transfusion 41:517521

5. Fielder PJ, Hass P, Nagel M, Stefanich E, Widmer R, Bennett GL, Keller GA, de Sauvage FJ, Eaton D 1997 Human platelets as a model for the binding and degradation of thrombopoietin. Blood 89:2782-2788

6. Li J, Xia Y, Kuter DJ 1999 Interaction of thrombopoietin with the platelet c-mpl receptor in plasma: binding, internalization, stability and pharmacokinetics. $\mathrm{Br} \mathrm{J}$ Haematol 106:345-356

7. Tahara T, Usuki K, Sato H, Ohashi H, Morita H, Tsumura H, Matsumoto A, Miyazaki H, Urabe A, Kato T 1996 A sensitive ELISA for measuring thrombopoietin in human serum: serum thrombopoietin levels in healthy volunteers and in patients with haematopoietic disorders. Br J Haematol 93:783-788

8. Emmons RVB, Reid DM, Cohen RL, Meng G, Young NS, Dunbar CE, Shulman NR 1996 Human thrombopoietin levels are high when thrombocytopenia is due to megakaryocyte deficiency and low when due to increased platelet destruction. Blood $87: 4068-4071$ 
9. Kosugi S, Kurata Y, Tomiyama Y, Tahara T, Kato T, Tadokoro S, Shiraga M, Honda S, Kanakura Y, Matsuzawa Y 1996 Circulating thrombopoietin level in chronic immune thrombocytopenic purpura. Br J Haematol 93:704-706

10. Porcelijn L, Folman CC, Bossers B, Huiskes E, Overbeeke MA, vd Schoot CE, de Haas M, von dem Borne AE 1998 The diagnostic value of thrombopoietin levels measurements in thrombocytopenia. Thromb Haemost 79:1101-1105

11. Cohen-Solal K, Vitrat N, Titeux M, Vainchenker W, Wendling F 1999 High-level expression of $\mathrm{Mpl}$ in platelets and megakaryocytes is independent of thrombopoietin. Blood 93:2859-2866

12. Mueller-Eckhardt C, Kiefel V, Grubert A, Kroll H, Weisheit M, Schmidt S, MuellerEckhardt G, Santoso S 1989348 cases of suspected neonatal alloimmune thrombocytopenia. Lancet 1:363-366

13. Williamson LM, Hackett G, Rennie J, Palmer CR, Maciver C, Hadfield R, Hughes D, Jobson S, Ouwehand WH 1998 The natural history of fetomaternal alloimmunization to the platelet-specific antigen HPA-1a (P1A1, Zwa) as determined by antenatal screening. Blood 92:2280-2287

14. von dem Borne AEGK, van Leeuwen EF, von Riesz LE, van Boxtel CJ, Engelfriet CP 1981 Neonatal alloimmune thrombocytopenia: detection and characterization of the responsible antibodies by the platelet immunofluorescence test. Blood 57:649-656

15. Warwick RM, Vaughan J, Murray N, Lubenko A, Roberts I 1994 In vitro culture of colony forming unit-megakaryocyte (CFU-MK) in fetal alloimmune thrombocytopenia. Br J Haematol 88:874-877

16. Bizzaro N, Dianese G 1988 Neonatal alloimmune amegakaryocytosis. Case report. Vox Sang 54:112-114

17. Tsukada T, Ueyama J, Miyakoshi S, Morinaga S, Mutoh Y, Yamaguchi H, Matsuya S 1990 [Diagnostic significance of the number of bone marrow megakaryocytes in cases with decreased platelet production evidenced by platelet kinetic study]. Rinsho Ketsueki 31:903-910
18. Bussel JB, Zabusky MR, Berkowitz RL, McFarland JG 1997 Fetal alloimmune thrombocytopenia. N Engl J Med 337:22-26

19. Kanhai HHH, Porcelijn L, van Zoeren D, Klumper F, Vietor H, Meerman RH, Brand A 1996 Antenatal care in pregnancies at risk of alloimmune thrombocytopenia: report of 19 cases in 16 families. Eur J Obstet Gynecol Reprod Biol 68:67-73

20. Folman CC, von dem Borne AEGKr, Rensink IHJAM, Gerritsen W, van der Schoot CE, de Haas M, Aarden L 1997 Sensitive measurements of thrombopoietin by a monoclonal antibody based sandwich enzyme-linked immunosorbent assay. Thromb Haemost 78:1262-1297

21. Sola MC, Juul SE, Meng YG, Garg S, Sims P, Calhoun DA, Dame JB, Christensen D 1999 Thrombopoietin (Tpo) in the fetus and neonate: Tpo concentrations in preterm and term neonates, and organ distribution of Tpo and its receptor (c-mpl) during human fetal development. Early Hum Dev 53:239-250

22. Ishiguro A, Nakahata T, Matsubara K, Hayashi Y, Kato T, Suzuki Y, Shimbo T 1999 Age-related changes in thrombopoietin in children: reference interval for serum thrombopoietin levels. Br J Haematol 106:884-888

23. Wolber EM, Dame C, Fahnenstich H, Hofmann D et al 1999 Expression of the thrombopoietin gene in human fetal and neonatal tissues. Blood 94:97-105

24. Kuwaki T, Hagiwara T, Yuki C, Kodama I, Kato T, Miyazaki H 1998 Quantitative analysis of thrombopoietin receptors on human megakaryocytes. FEBS Lett 427:46-50

25. Harker LA, Marzec UM, Novembre F, Sundell IB, Waller EK, Karpatkin S, McClure HM, Kelly AB, Stead RB 1998 Treatment of thrombocytopenia in chimpanzees infected with human immunodeficiency virus by pegylated recombinant human megakaryocyte growth and development factor. Blood 91:4427-4433

26. Harker LA 1999 Physiology and clinical applications of platelet growth factors. Curr Opin Hematol 6:127-134 